\title{
Computational Experience with Rigorous Error Bounds for the Netlib Linear Programming Library
}

\author{
Christian Keil (c.keil@tu-harburg.de) and Christian Jansson \\ (jansson@tu-harburg.de) \\ Technical University Hamburg-Harburg
}

\begin{abstract}
The Netlib library of linear programming problems is a well known suite containing many real world applications. Recently it was shown by Ordóñez and Freund that $71 \%$ of these problems are ill-conditioned. Hence, numerical difficulties may occur. Here, we present rigorous results for this library that are computed by a verification method using interval arithmetic. In addition to the original input data of these problems we also consider interval input data. The computed rigorous bounds and the performance of the algorithms are related to the distance to the next ill-posed linear programming problem.
\end{abstract}

Keywords: linear programming, interval arithmetic, rigorous error bounds, sensitivity analysis, Netlib linear programming library

AMS Subject classification: 90C05, 65G30, 65N15

\section{Introduction}

The Netlib suite of linear optimization problems [15] includes many real world applications like stochastic forestry problems, oil refinery problems, flap settings of aircraft, pilot models, audit staff scheduling, truss structure problems, airline schedule planning, industrial production and allocation models, image restoration problems, and multisector economic planning problems. It contains problems ranging in size from 32 variables and 27 constraints up to 15695 variables and 16675 constraints.

In a recent publication Ordóñez and Freund have shown $71 \%$ of the Netlib problems to be ill-conditioned [20]. Only $19 \%$ of the problem instances remain ill-conditioned after preprocessing techniques are applied. In a paper by Fourer and Gay [5], however, it is observed that rounding errors in presolve may change the status of a linear program from feasible to infeasible, and vice versa.

The goal of this paper is to present verified numerical results for both the unprocessed original input data of this library and interval input data. These results are obtained by a verification method for linear programming problems that is presented in [9]. Our central observation is that, roughly spoken, rigorous error bounds together with certificates of feasibility are obtained for the well-posed problem instances. Further-

(C) 2005 Kluwer Academic Publishers. Printed in the Netherlands. 
more, for many ill-posed problem instances a rigorous lower or upper bound of the optimal value can be computed. The computational costs increase with decreasing distances to primal or dual infeasibility, that is with decreasing distance to ill-posedness.

We mention that the algorithms used to compute these rigorous bounds are not only useful for linear programming problems. They can also be used in global optimization and mixed-integer programming whenever linear relaxations must be solved (see for example Floudas [4] and Neumaier [18]). Thus safe results can be obtained for these nonlinear problems.

This paper is organized as follows. Section 2 contains the basic theorems of our verification method. In Section 3 a brief introduction to condition numbers for linear programming problems is given. The numerical results of our computations are discussed in Section 4. Finally, in Section 5, some concluding remarks are given. The tables with the numerical results are presented in the appendix.

\section{Rigorous Error Bounds}

We consider the linear programming problem

$$
\begin{gathered}
f^{*}:=\min _{x \in X} c^{T} x \\
X:=\left\{x \in \mathbb{R}^{n}: A x \leq a, B x=b, \underline{x} \leq x \leq \bar{x}\right\},
\end{gathered}
$$

with $f^{*}$ becoming $+\infty$ if $X$ is empty.

The input parameter are

$$
P=(A, B, a, b, c) \in \mathbb{R}^{(m+p+1) n+m+p},
$$

where $A$ is a real $m \times n$ matrix, $\mathrm{B}$ a real $p \times n$ matrix, $c, x \in \mathbb{R}^{n}$, $a \in \mathbb{R}^{m}$, and $b \in \mathbb{R}^{p}$. Further, the simple bounds $\underline{x} \leq \bar{x}$, which may be infinite; that is $\underline{x}_{j}:=-\infty$ or $\bar{x}_{j}:=+\infty$ for some $j \in\{1, \ldots, n\}$. The set of indices where the simple bounds are both infinite is denoted by

$$
J^{\infty}:=\left\{j \in\{1, \ldots, n\}: \underline{x}_{j}=-\infty, \text { and } \bar{x}_{j}=+\infty\right\}
$$

and its complement by $J^{r}:=\{1, \ldots, n\} \backslash J^{\infty}$.

The input data may be uncertain. We describe these uncertainties by considering a family of linear programming problems $P$, where $P \in \mathbf{P}$ and $\mathbf{P}$ represents the corresponding interval quantities. We require only some elementary facts about interval arithmetic, which can be found for example in Alefeld and Herzberger [1], Hansen and Walster [7], Kearfott [10], Moore [14], and Neumaier [16], [17]. 
To compute a rigorous upper bound, the basic idea is to determine an interval vector $\mathbf{x}$ that contains a feasible solution for every $P \in \mathbf{P}$, being in the relative interior of $X$. This solution should be close to an optimal solution but sufficiently far away from degeneracy and infeasibility. The next theorem gives favourable characteristics of $\mathbf{x}$.

THEOREM 1. Let $\mathbf{P}:=(\mathbf{A}, \mathbf{B}, \mathbf{a}, \mathbf{b}, \mathbf{c})$ be a family of lp-problems with input data $P \in \mathbf{P}$ and simple bounds $\underline{x} \leq \bar{x}$. Suppose that there exists an interval vector $\mathbf{x} \in \mathbb{I}^{n}$ such that

$$
\mathbf{A x} \leq \mathbf{a}, \quad \underline{x} \leq \mathbf{x} \leq \bar{x},
$$

and

$$
\forall B \in \mathbf{B}, b \in \mathbf{b} \exists x \in \mathbf{x}: B x=b .
$$

Then for every $P \in \mathbf{P}$ there exists a primal feasible solution $x(P) \in \mathbf{x}$, and the inequality

$$
\sup _{P \in \mathbf{P}} f^{*}(P) \leq \overline{f^{*}}:=\max \left\{\mathbf{c}^{T} \mathbf{x}\right\}
$$

is satisfied. Moreover, if the objective function is bounded from below for every lp-problem with input data $P \in \mathbf{P}$, then each problem has an optimal solution.

The following theorem provides the basic characteristics of a rigorous lower bound.

THEOREM 2. Let $\mathbf{P}:=(\mathbf{A}, \mathbf{B}, \mathbf{a}, \mathbf{b}, \mathbf{c})$ be a family of lp-problems with input data $P \in \mathbf{P}$ and simple bounds $\underline{x} \leq \bar{x}$. Suppose that there exist interval vectors $\mathbf{y} \in \mathbb{R}^{m}$ and $\mathbf{z} \in \mathbb{R}^{p}$ such that

(i) the sign condition

$$
\mathbf{y} \leq 0
$$

holds true,

(ii) for $j \in J^{\infty}$ the equations

$$
\begin{gathered}
\forall A \in \mathbf{A}, B \in \mathbf{B}, c \in \mathbf{c} \exists y \in \mathbf{y}, z \in \mathbf{z}: \\
\left(A_{: j}\right)^{T} y+\left(B_{: j}\right)^{T} z=c_{j}
\end{gathered}
$$

are fulfilled,

(iii) and for $j \in J^{r}$ the intervals

$$
\mathbf{d}_{j}:=\mathbf{c}_{j}-\left(\mathbf{A}_{: j}\right)^{T} \mathbf{y}-\left(\mathbf{B}_{: j}\right)^{T} \mathbf{z}
$$

satisfy the inequalities

$$
\begin{aligned}
& \mathbf{d}_{j} \leq 0, \text { if } \underline{x}_{j}=-\infty \\
& \mathbf{d}_{j} \geq 0, \text { if } \bar{x}_{j}=+\infty .
\end{aligned}
$$


Then the inequality

$$
\inf _{P \in \mathbf{P}} f^{*}(P) \geq \underline{f^{*}}:=\min \left\{\mathbf{a}^{T} \mathbf{y}+\mathbf{b}^{T} \mathbf{z}+\sum_{\substack{j \in J^{r} \\ \overline{\mathbf{d}}_{\mathbf{j}}>0}} \underline{x}_{j} \mathbf{d}_{j}^{+}+\sum_{\substack{j \in J^{r} \\ \underline{\mathbf{d}}_{\mathbf{j}}<0}} \bar{x}_{j} \mathbf{d}_{j}^{-}\right\}
$$

is fulfilled, and $f^{*}$ is a finite lower bound of the global minimum value. Moreover, if

(a) all input data are point data (i.e. $P=\mathbf{P})$,

(b) $P$ has an optimal solution $\left(y^{*}, z^{*}, u^{*}, v^{*}\right)$,

(c) $\mathbf{y}:=y^{*}, \mathbf{z}:=z^{*}$,

(d) the quantities in (3) and (4) are calculated exactly,

then the conditions (i),(ii) and (iii) are satisfied, and the optimal value $f^{*}(P)=f^{*}$; that is, this lower error bound is sharp for point input data and exact computations.

For proofs of these two theorems as well as algorithms for computing appropriate interval vectors $\mathbf{x}, \mathbf{y}$, and $\mathbf{z}$ the reader is referred to [9]. Roughly spoken, the algorithms try to find these interval vectors in an iterative manner by computing approximate solutions of perturbed linear programming problems. In the special case where all simple bounds are finite the conditions (ii) and (iii) of Theorem 2 are trivially satisfied. Hence, for each nonnegative interval vector $\mathbf{y}$ the right hand side of (4) delivers a rigorous lower bound in $O\left(n^{2}\right)$ operations.

We emphasize that the previous analysis gives a rigorous certificate for the existence of optimal solutions if both bounds $f^{*}$ and $\overline{f^{*}}$ are finite. Our numerical experience is that these bounds can be computed also for degenerate and ill-conditioned problems provided the linear programming solver has computed sufficiently accurate results. The quality of $f^{*}$ and $\overline{f^{*}}$ depends mainly on the accuracy of the computed approximations.

A generalization of our approach to convex problems is described in [8].

In [11] the algorithms presented in [9] were implemented using the interval library PROFIL/BIAS [12]. There the reader can find primary numerical results for the Netlib suite. The linear programming solver used to compute approximate solutions was lp_solve [3].

Recently, Neumaier and Shcherbina [19] have investigated rigorous error bounds for mixed-integer linear programming problems. In their paper, in addition to rigorous cuts and a certificate of infeasibility, a rigorous lower bound for linear programming problems with exact input 
data and finite simple bounds is presented. Our focus is on problems with uncertain input data and simple bounds which may be infinite. In the overlapping part of both papers, where the simple bounds are finite, both rigorous lower bounds coincide.

Beeck [2], Krawzcyk [13], and Rump [21] have developed methods for computing rigorous error bounds for lp-problems where the optimal solution is unique. They use the simplex method for the computation of an optimal basic index set. Then with interval methods the optimality of this index set is verified a posteriori, and rigorous error bounds for the optimal vertex and the optimal value are calculated. These methods are more expensive, they require $O\left(n^{3}\right)$ operations even for finite simple bounds, and they can only be applied to non-degenerate problems. Furthermore, the distances to primal and dual infeasibility must be greater than 0 . Since most problems of the Netlib lp library do not have these properties, the mentioned methods do not work.

\section{Condition Numbers}

In order to define the condition number for linear programming problems, Ordóñez and Freund used the ground-set format

$$
\begin{gathered}
f^{*}(P):=\min _{x \in X(P)} c^{T} x \\
X(P):=\left\{x \in \mathbb{R}^{n}: A x-b \in C_{Y}, x \in S\right\} .
\end{gathered}
$$

A specific ground-set problem is defined by its input data $P=$ $(A, b, c)$ consisting of the real $m \times n$ matrix $A$ and the real vectors $b \in \mathbb{R}^{m}$ and $c \in \mathbb{R}^{n}$. The set $C_{Y} \subseteq \mathbb{R}^{m}$ is a closed convex cone, and the set $S \subseteq \mathbb{R}^{n}$ is closed and convex.

The corresponding dual problem is

$$
\begin{gathered}
f^{*}(P):=\max _{(y, v) \in Y(P)} b^{T} y-v \\
Y(P):=\left\{(y, v) \in \mathbb{R}^{m \times n}:\left(c-A^{T} y, v\right) \in C_{S}^{*}, y \in C_{Y}^{*}\right\} .
\end{gathered}
$$

Here, $C_{Y}^{*}$ denotes the dual cone of $C_{Y}$, i.e.

$$
C_{Y}^{*}:=\left\{y \in \mathbb{R}^{m}: z^{T} y \geq 0 \text { for all } z \in C_{Y}\right\},
$$

and $C_{S}^{*}$ denotes the dual cone of

$$
C_{S}:=\{(x, t): x \in t S \text { and } t>0\} .
$$

The distances to primal and dual infeasibility are defined by

$$
\begin{aligned}
& \rho_{p}(P):=\inf \{\|\Delta P\|: X(P+\Delta P)=\emptyset\} \\
& \rho_{d}(P):=\inf \{\|\Delta P\|: Y(P+\Delta P)=\emptyset\},
\end{aligned}
$$


where $\Delta P:=(\Delta A, \Delta b, \Delta c)$ and

$$
\|\Delta P\|:=\max \left\{\|\Delta A\|,\|\Delta b\|_{1},\|\Delta c\|_{\infty}\right\},
$$

with $\|\Delta A\|$ denoting the corresponding operator norm.

Using this choice of norms, Ordóñez and Freund have shown that the distances to infeasibility can be computed by solving $2 n+2 m$ linear programming problems of size roughly that of the original problem.

The condition number of a linear programming problem is defined as the quotient of the norm of the input data and the smallest distance to infeasibility,

$$
\operatorname{cond}(P):=\frac{\|P\|}{\min \left\{\rho_{p}(P), \rho_{d}(P)\right\}} .
$$

A problem is called ill-posed if $\min \left\{\rho_{p}(P), \rho_{d}(P)\right\}=0$ or equivalently $\operatorname{cond}(P)=\infty$.

We mention that Ordóñez and Freund have computed the condition numbers for the problems in the Netlib lp library, but not rigorously.

Our linear programming format (1) can be described in the groundset format by aggregating the equality and inequality constraints to

$$
\left(\begin{array}{l}
A \\
B
\end{array}\right) x-\left(\begin{array}{l}
a \\
b
\end{array}\right) \in C_{Y}:=\left(\begin{array}{c}
\mathbb{R}_{-}^{m} \\
0
\end{array}\right)
$$

and using $S:=\left\{x \in \mathbb{R}^{n}: \underline{x} \leq x \leq \bar{x}\right\}$. This transformation yields the condition number for our format.

\section{Numerical Results}

In the following we present our rigorous results for the Netlib suite of linear programming problems. The implementation is a slight modification of the algorithms in [9] with respect to the deflation parameter vector $\varepsilon$. The linear programming solver used to compute approximate solutions was lp_solve 5.5 [3]. Compiler suite was the gcc version 3.3.1 [6]. All computations were done on a PC with $2.8 \mathrm{GHz}$.

In order to compare the results, we have chosen exactly the set of problems that Ordóñez and Freund [20] have computed condition numbers for. For the problems DEGEN3 and PILOT, lp_solve was aborted because the original problem had not been solved after 24 hours, leaving 87 problems in the test set.

Our results are displayed in the appendix. Summarizing, it can be seen that in almost all cases rigorous upper bounds $\overline{f^{*}}$ and rigorous 
lower bounds $f^{*}$ are computed if the distance to respectively primal infeasibility $\rho_{p}$ and dual infeasibility $\rho_{d}$ is greater than 0 . Rigorous bounds and a certificate of the existence of optimal solutions are obtained for well-posed problems.

Table I shows the accuracy of the rigorous bounds. The first column contains the name of the problem. Columns two and three contain the distances to infeasibility $\rho_{d}$ and $\rho_{p}$ as computed by Ordóñez and Freund. Then the lower and upper bound rounded to 5 decimal digits of precision are displayed, and the last column contains the relative error

$$
\mu:=\frac{\mid \overline{f^{*}}-\underline{f^{*} \mid}}{\max \left\{1,0.5\left(\left|\overline{f^{*} \mid}+\right| \underline{f^{*} \mid} \mid\right)\right\}} .
$$

If one of the bounds in the quotient $\mu$ could not be computed, it is substituted by the approximate optimal value delivered by lp_solve. In the case of both bounds being infinite, $\mu$ is set to NaN (i.e., Not a Number).

Throughout our experiments we have used lp_solve 5.5 with only the default optimization parameters. From Table I it can be seen that for almost all problems the relative error $\mu$ varies between $1 \cdot 10^{-8}$ and $1 \cdot 10^{-16}$. With lp_solve's default stopping tolerance of $1 \cdot 10^{-9}$, this is about the best one could expect.

We see that in almost all cases the rigorous lower and upper bound is finite if the distance to dual and primal infeasibility is greater than 0 , respectively. Only the problems SCSD 8 and SCTAP1 deliver no upper bound despite a primal distance to infeasibility greater than 0 . On the other hand, the problems 25FV47, 80BAU3B, BEACONFD, BNL2, $C Y C L E$, D2Q06C, E226, RECIPE, SCRS8, STANDGUB deliver a lower bound and 80BAU3B, ADLITTLE, E226, FINNIS, GFRD-PNC, $S C 105, S C 205, S C 50 A, S C 50 B$ an upper one although the corresponding distance to infeasibility is equal to 0 . We guess that for these problems the distances to infeasibility given in the paper of Ordóñez and Freund [20] are in fact not zero. This may result from computing them numerically without verification.

The large relative errors $\mu$ for the problems SCSD6, SCTAP2, SCTAP 3 are due to the bad upper bounds. We hope to improve this in the future.

Table II shows that although almost all problems have unbounded variables, in many cases the rigorous lower bound can be computed within a fraction of the computational work that is required for solving the problem approximately. There $i_{f^{*}}, i_{f^{*}}$ denote the number of iterations for computing the lower and upper bound respectively and $t_{f^{*}} / t_{f^{*}}, t_{\overline{f^{*}}} / t_{f^{*}}$ denote the corresponding time ratios. If this ratio can 
not be computed due to problems being solved faster than the timer resolution of $0.01 \mathrm{sec}$, this column is left empty. Only the problems FIT1D, FIT2D, SIERRA have finite simple bounds yielding an infinite distance to dual infeasibility. This results in a lower bound without the need of iterating. The problem RECIPE also delivers a lower bound without any iterations, and 65 problems deliver a lower bound in only 1 iteration. The huge time ratios for problems $A G G, B E A C O N F D$, and $S C R S 8$ stem from lp_solve timing out after 24 hours of trying to solve a perturbed problem.

Infinite error bounds for the optimal value result from ill-posedness and are expressed by exceeding iteration counts, rank deficient constraint matrices, or in five cases, by numerical problems during the solution of perturbed linear programs. Table II shows that the determination of an infinite bound is very time consuming if the iteration count exceeds the set limit of 31 .

Since PROFIL/BIAS does not support sparse structures, the memory usage increases dramatically when transforming the constraint matrices from lp_solve's sparse representation to PROFIL/BIAS's nonsparse one. The usage of non-sparse interval linear solvers adds to this effect. This is the reason why in some cases even few iterations result in large time ratios. In the future, we will enhance PROFIL/BIAS to support sparse structures.

We emphasize that the bounds depend drastically on the used lpsolver. Even in the case where we used the same solver but a previous version (lp_solve 3.2) the results got worse. Some rigorous lower bounds computed with lp_solve 3.2 are listed in Table III. We mention that in all cases the approximations computed by lp_solve are within the rigorous bounds.

To test the quality of the algorithms when using interval data, the problem parameters of the Netlib lp problems were multiplied by the interval $\left[1-10^{-6}, 1+10^{-6}\right]$ yielding a relative uncertainty. Table IV contains the error bounds for these problems with interval parameters, and Table $\mathrm{V}$ shows the performance. Compared with the radius $r=1 \cdot 10^{-6}$ of the interval input data, the algorithms give in most cases very accurate worst case bounds. The huge time ratios for $A G G$, $B E C O N F D, F F F F F 800$ and PILOT.WE originate again from lp_solve timeouts while trying to solve perturbed problems.

\section{Concluding Remarks}

Computing rigorous error bounds for the optimal value of linear programming problems together with a certificate of the existence of opti- 
mal solutions has been shown to be possible in various areas of applications.

These bounds can also be used in global optimization and mixedinteger nonlinear programming whenever linear relaxations must be solved in branch and bound algorithms.

In future implementations we plan to incorporate several improvements like sparse structures in PROFIL/BIAS, other linear programming solvers, other deflation parameters, and a rigorous preprocessing of the data.

\section{Appendix}

\section{A. Tables}

Table I.: Rigorous bounds for the Netlib problems

$\rho_{d}$ - distance to dual infeasibility, $\rho_{p}$ - distance to primal infeasibility,

$f^{*}$ - lower bound, $\overline{f^{*}}$ - upper bound, $\mu$ - relative accuracy

\begin{tabular}{|c|c|c|c|c|c|}
\hline Name & $\rho_{d}$ & $\rho_{p}$ & $f^{*}$ & $\overline{f^{*}}$ & $\mu$ \\
\hline $25 \mathrm{FV} 47$ & 0 & 0 & $5.5018 e+\overline{03}$ & $\infty$ & $8.5111 e-08$ \\
\hline 80BAU3B & 0 & 0 & $9.8722 e+05$ & $9.8722 e+05$ & $5.6653 e-08$ \\
\hline ADLITTLE & 0.051651 & 0 & $2.2549 e+05$ & $2.2549 e+05$ & $3.6470 e-08$ \\
\hline AFIRO & 1.000000 & 0.397390 & $-4.6475 e+02$ & $-4.6475 e+02$ & $2.0481 e-08$ \\
\hline AGG2 & 0.771400 & 0 & $-2.0239 e+07$ & $\infty$ & $2.0868 e-08$ \\
\hline AGG3 & 0.771400 & 0 & $1.0312 e+07$ & $\infty$ & $7.3998 e-08$ \\
\hline AGG & 0.771400 & 0 & $-3.5992 e+07$ & $\infty$ & $2.7323 e-08$ \\
\hline BANDM & 0.000418 & 0 & $-1.5863 e+02$ & $\infty$ & $7.0742 e-08$ \\
\hline BEACONFD & 0 & 0 & $3.3592 e+04$ & $\infty$ & $9.9997 e-09$ \\
\hline BLEND & 0.040726 & 0.003541 & $-3.0812 e+01$ & $-3.0812 e+01$ & $1.3560 e-07$ \\
\hline BNL1 & 0.106400 & 0 & $1.9776 e+03$ & $\infty$ & $7.2244 e-08$ \\
\hline BNL2 & 0 & 0 & $1.8112 e+03$ & $\infty$ & $2.0899 e-08$ \\
\hline BORE3D & 0.003539 & 0 & $1.3731 e+03$ & $\infty$ & $1.3362 e-08$ \\
\hline BRANDY & 0 & 0 & $-\infty$ & $\infty$ & $\mathrm{NaN}$ \\
\hline CAPRI & 0.095510 & 00252 & $2.6900 e+03$ & $2.6900 e+03$ & $1.6905 e-07$ \\
\hline CYCLE & 0 & 0 & $-5.2264 e+00$ & $\infty$ & $1.4574 e-08$ \\
\hline CZPROB & 0.008807 & 0 & $2.1852 e+06$ & $\infty$ & $1.0915 e-08$ \\
\hline D2Q06C & 0 & 0 & $1.2278 e+05$ & $\infty$ & $4.5242 e-08$ \\
\hline D6CUBE & 2.000000 & 0 & $3.1549 e+02$ & $\infty$ & $1.6796 e-08$ \\
\hline DEGEN2 & 1.000000 & 0 & $-1.4352 e+03$ & $\infty$ & $9.3150 e-09$ \\
\hline E226 & 0 & 0 & $-2.5865 e+01$ & $-2.5865 e+01$ & $9.1411 e-08$ \\
\hline ETAMACRO & 0.200000 & 0 & $-7.5572 e+02$ & $\infty$ & $4.4004 e-09$ \\
\hline FFFFF800 & 0.033046 & 0 & $5.5568 e+05$ & $\infty$ & $4.1052 e-08$ \\
\hline FINNIS & 0 & 0 & $-\infty$ & $1.7279 e+05$ & $4.8378 e-08$ \\
\hline FIT1D & $\infty$ & 3.500000 & $-9.1464 e+03$ & $-9.1464 e+03$ & $6.1900 e-09$ \\
\hline FIT1P & 0.437500 & 1.271887 & $9.1464 e+03$ & $9.1464 e+03$ & $1.1418 e-08$ \\
\hline FIT2D & $\infty$ & 17.000000 & $-6.8464 e+04$ & $-6.8464 e+04$ & $4.8472 e-09$ \\
\hline FIT2P & .000000 & 1.057333 & $6.8464 e+04$ & $6.8464 e+04$ & $6.9205 e-09$ \\
\hline GANGES & 1.000000 & 0 & $-1.0959 e+05$ & $\infty$ & $3.5123 e-09$ \\
\hline GFRD-PNC & 0.347032 & 0 & $6.9022 e+06$ & $6.9022 e+06$ & $5.5746 e-08$ \\
\hline GREENBEA & 0 & 0 & $-\infty$ & $\infty$ & $\mathrm{NaN}$ \\
\hline GREENBEB & 0 & 0 & $-\infty$ & $\infty$ & $\mathrm{NaN}$ \\
\hline GROW15 & 968073 & 2842 & $-1.0687 e+08$ & $-1.0687 e+08$ & $3.5135 e-09$ \\
\hline GROW22 & 0.968073 & 0.572842 & $-1.6083 e+08$ & $-1.6083 e+08$ & $3.7475 e-09$ \\
\hline GROW7 & 0.968073 & 0.572842 & $-4.7788 e+07$ & $-4.7788 e+07$ & $3.6032 e-09$ \\
\hline ISRAEL & 0.166850 & 0.027248 & $-8.9664 e+05$ & $-8.9664 e+05$ & $1.5935 e-08$ \\
\hline KB2 & 0.018802 & 0.000201 & $-1.7499 e+03$ & $-1.7499 e+03$ & $2.1792 e-08$ \\
\hline LOTFI & 0 & 0.000306 & $-\infty$ & $-2.5265 e+01$ & $4.5049 e-09$ \\
\hline MAROS & 0 & 0 & $-\infty$ & $\infty$ & $\mathrm{NaN}$ \\
\hline MAROS-R7 & 0.628096 & 1.000000 & $1.4972 e+06$ & $1.4972 e+06$ & $8.5236 e-09$ \\
\hline MODSZK1 & 0.108469 & 0 & $3.2057 e+02$ & $\infty$ & $1.5512 e-04$ \\
\hline PEROLD & 0.000943 & 0 & $-9.3808 e+03$ & $\infty$ & $2.2012 e-08$ \\
\hline PILOT4 & 0.000075 & 0 & $-2.5811 e+03$ & $\infty$ & $2.8098 e-08$ \\
\hline PILOT 87 & 0 & 0 & $-\infty$ & $\infty$ & $\mathrm{NaN}$ \\
\hline
\end{tabular}




\begin{tabular}{|c|c|c|c|c|c|}
\hline Name & $\rho_{d}$ & $\rho_{p}$ & $f^{*}$ & $\overline{\overline{f^{*}}}$ & $\mu$ \\
\hline PILOT.JA & 0.000750 & 0 & $-6.1131 e+\overline{03}$ & $\infty$ & $1.5904 e-08$ \\
\hline PILOTNOV & 0.000750 & 0 & $-4.4973 e+03$ & $\infty$ & $3.2619 e-08$ \\
\hline PILOT.WE & 0.044874 & 0 & $-2.7201 e+06$ & $\infty$ & $5.2748 e-08$ \\
\hline QAP8 & 4.000000 & 0 & $2.0350 e+02$ & $\infty$ & $5.2913 e-08$ \\
\hline RECIPE & 0 & 0 & $-2.6662 e+02$ & $\infty$ & $4.2641 e-16$ \\
\hline SC105 & 0.133484 & 0 & $-5.2202 e+01$ & $-5.2202 e+01$ & $7.7626 e-08$ \\
\hline $\mathrm{SC} 205$ & 0.010023 & 0 & $-5.2202 e+01$ & $-5.2202 e+01$ & $9.0740 e-08$ \\
\hline SC50A & 0.562500 & 0 & $-6.4575 e+01$ & $-6.4575 e+01$ & $5.6764 e-08$ \\
\hline SC50B & 0.421875 & 0 & $-7.0000 e+01$ & $-7.0000 e+01$ & $5.7599 e-08$ \\
\hline SCAGR25 & 0.034646 & 0.021077 & $-1.4753 e+07$ & $-1.4753 e+07$ & $3.7821 e-08$ \\
\hline SCAGR7 & 0.034646 & 0.022644 & $-2.3314 e+06$ & $-2.3314 e+06$ & $3.9152 e-08$ \\
\hline SCFXM1 & 0 & 0 & $-\infty$ & $\infty$ & $\mathrm{NaN}$ \\
\hline SCFXM2 & 0 & 0 & $-\infty$ & $\infty$ & $\mathrm{NaN}$ \\
\hline SCFXM3 & 0 & 0 & $-\infty$ & $\infty$ & $\mathrm{NaN}$ \\
\hline SCORPION & 0.949393 & 0 & $1.8781 e+03$ & $\infty$ & $2.7948 e-08$ \\
\hline SCRS8 & 0 & 0 & $9.0430 e+02$ & $\infty$ & $3.4248 e-08$ \\
\hline SCSD1 & 1.000000 & 5.037757 & $8.6667 e+00$ & $8.6668 e+00$ & $1.0579 e-05$ \\
\hline SCSD6 & 1.000000 & 1.603351 & $5.0500 e+01$ & $5.0707 e+01$ & $4.0917 e-03$ \\
\hline SCSD8 & 1.000000 & 0.268363 & $9.0500 e+02$ & $\infty$ & $6.3831 e-08$ \\
\hline SCTAP1 & 1.000000 & 0.032258 & $1.4122 e+03$ & $\infty$ & $2.1640 e-08$ \\
\hline SCTAP2 & 1.000000 & 0.586563 & $1.7248 e+03$ & $1.9777 e+03$ & $1.3662 e-01$ \\
\hline SCTAP3 & 1.000000 & 0.381250 & $1.4240 e+03$ & $2.0866 e+03$ & $3.7748 e-01$ \\
\hline SHARE1B & 0.000751 & 0.000015 & $-7.6589 e+04$ & $-7.6589 e+04$ & $1.7119 e-07$ \\
\hline SHARE2B & 0.287893 & 0.001747 & $-4.1573 e+02$ & $-4.1573 e+02$ & $4.0674 e-07$ \\
\hline SHELL & 1.777778 & 0 & $1.2088 e+09$ & $\infty$ & $4.6203 e-09$ \\
\hline SHIP04L & 13.146000 & 0 & $1.7933 e+06$ & $\infty$ & $9.7665 e-09$ \\
\hline SHIP04S & 13.146000 & 0 & $1.7987 e+06$ & $\infty$ & $1.0115 e-08$ \\
\hline SHIP08L & 21.210000 & 0 & $1.9091 e+06$ & $\infty$ & $1.0593 e-08$ \\
\hline SHIP08S & 21.210000 & 0 & $1.9201 e+06$ & $\infty$ & $1.1197 e-08$ \\
\hline SHIP12L & 7.434000 & 0 & $1.4702 e+06$ & $\infty$ & $1.1950 e-08$ \\
\hline SHIP12S & 7.434000 & 0 & $1.4892 e+06$ & $\infty$ & $1.3700 e-08$ \\
\hline SIERRA & $\infty$ & 0 & $1.5394 e+07$ & $\infty$ & $5.3601 e-14$ \\
\hline STAIR & 0 & 0.000580 & $-\infty$ & $-2.5127 e+02$ & $5.4796 e-09$ \\
\hline STANDATA & 1.000000 & 0 & $1.2577 e+03$ & $\infty$ & $1.2619 e-08$ \\
\hline STANDGUB & 0 & 0 & $1.2577 e+03$ & $\infty$ & $1.2619 e-08$ \\
\hline STANDMPS & 1.000000 & 0 & $1.4060 e+03$ & $\infty$ & $1.3776 e-08$ \\
\hline STOCFOR1 & 0.011936 & 0.001203 & $-4.1132 e+04$ & $-4.1132 e+04$ & $4.2148 e-08$ \\
\hline STOCFOR2 & 0.000064 & 0.000437 & $-3.9024 e+04$ & $-3.9024 e+04$ & $5.6996 e-08$ \\
\hline TRUSS & 10.000000 & 0.518928 & $4.5882 e+05$ & $4.5882 e+05$ & $2.3769 e-06$ \\
\hline TUFF & 0.017485 & 0 & $2.8677 e-01$ & $\infty$ & $5.3744 e-03$ \\
\hline VTP.BASE & 0.500000 & 0 & $1.2983 e+05$ & $\infty$ & $3.4508 e-08$ \\
\hline WOOD1P & 1.000000 & 0 & $1.4429 e+00$ & $\infty$ & $4.3361 e-08$ \\
\hline WOODW & 1.000000 & 0 & $1.3045 e+00$ & $\infty$ & $2.4401 e-08$ \\
\hline
\end{tabular}

Table II.: Performance of the Netlib bounds

$i_{f *}$ - iterations to compute lower bound,

$\overline{t_{f^{*}}}$ - time to compute lower bound, $t_{f^{*}}-$ time to compute approximate solution,

$i \overline{\overline{f^{*}}}-$ iterations to compute upper bound,

$t \frac{f^{*}}{f^{*}}$-time to compute upper bound

\begin{tabular}{lrrrr}
\hline Name & $i_{f^{*}}$ & $t_{f^{*} / t_{f} *}$ & $i \overline{f^{*}}$ & \multicolumn{1}{c}{$\bar{f}^{*} / t_{f^{*}}$} \\
\hline 25FV47 & 1 & 0.102 & 0 & 0.030 \\
80BAU3B & 1 & 1.943 & 3 & 0.808 \\
ADLITTLE & 1 & 0.000 & 4 & 0.000 \\
AFIRO & 1 & & 5 & \\
AGG2 & 1 & 1.000 & 31 & 7.667 \\
AGG3 & 1 & 0.750 & 31 & 5.750 \\
AGG & 1 & 0.500 & 2 & 4320000.000 \\
BANDM & 1 & 0.300 & 31 & 58.000 \\
BEACONFD & 1 & 1.000 & 12 & 8640100.000 \\
BLEND & 1 & 0.000 & 5 & 1.000 \\
\hline BNL1 & 1 & 0.519 & 0 & 3.444 \\
BNL2 & 3 & 2.648 & 31 & 197.643 \\
BORE3D & 1 & 1.000 & 0 & 11.000 \\
BRANDY & 31 & 2.000 & 0 & 0.000 \\
CAPRI & 1 & 0.333 & 4 & 9.333 \\
CYCLE & 10 & 2.574 & 0 & 1.626 \\
CZPROB & 1 & 0.469 & 31 & 68.531 \\
D2Q06C & 1 & 0.277 & 31 & 26.499 \\
D6CUBE & 1 & 0.345 & 0 & 7.480 \\
DEGEN2 & 1 & 0.152 & 0 & 1.273 \\
\hline E226 & 1 & 0.167 & 4 & 0.500 \\
ETAMACRO & 3 & 0.636 & 0 & 6.182 \\
& continued....
\end{tabular}


Computational Experience with Rigorous Error Bounds for the Netlib lp Library 11

\begin{tabular}{|c|c|c|c|c|}
\hline Name & $i_{f *}$ & $t_{f *} / t_{f} *$ & $i \overline{f^{*}}$ & $\overline{t_{f^{*}}} / t_{f^{*}}$ \\
\hline FFFFF800 & $\overline{11}$ & 0.421 & 31 & 35.000 \\
\hline FINNIS & 31 & 3.500 & 2 & 2.250 \\
\hline FIT1D & 0 & 0.000 & 2 & 0.125 \\
\hline FIT1P & 1 & 0.321 & 10 & 68.887 \\
\hline FIT2D & 0 & 0.003 & 1 & 0.009 \\
\hline FIT2P & 1 & 0.722 & 12 & 243.487 \\
\hline GANGES & 1 & 0.841 & 31 & 620.455 \\
\hline GFRD-PNC & 1 & 0.833 & 7 & 173.417 \\
\hline GREENBEA & 31 & 0.773 & 0 & 9.911 \\
\hline GREENBEB & 31 & 0.813 & 0 & 10.568 \\
\hline GROW15 & 1 & 0.051 & 6 & 4.966 \\
\hline GROW22 & 1 & 0.060 & 9 & 13.667 \\
\hline GROW7 & 1 & 0.000 & 10 & 4.667 \\
\hline ISRAEL & 1 & 0.000 & 1 & 0.333 \\
\hline KB2 & 1 & & 4 & \\
\hline LOTFI & 31 & 2.000 & 5 & 5.500 \\
\hline MAROS & 31 & 0.919 & 0 & 2.364 \\
\hline MAROS-R7 & 1 & 0.381 & 10 & 105.517 \\
\hline MODSZK1 & 1 & 1.235 & 0 & 18.118 \\
\hline PEROLD & 4 & 0.573 & 31 & 16.183 \\
\hline PILOT4 & 3 & 0.680 & 31 & 22.080 \\
\hline PILOT 87 & 9 & 674.546 & 2 & 979.242 \\
\hline PILOT.JA & 3 & 0.330 & 0 & 0.378 \\
\hline PILOTNOV & 1 & 0.436 & 0 & 1.154 \\
\hline PILOT.WE & 1 & 0.141 & 6 & 9.559 \\
\hline QAP8 & 10 & 21.895 & 0 & 0.091 \\
\hline RECIPE & 0 & 0.000 & 0 & 0.000 \\
\hline $\mathrm{SC} 105$ & 1 & 0.000 & 1 & 0.000 \\
\hline SC205 & 1 & 0.500 & 1 & 2.000 \\
\hline SC50A & 1 & & 1 & \\
\hline SC50B & 1 & 0.000 & 1 & 0.000 \\
\hline SCAGR25 & 1 & 0.308 & 4 & 27.769 \\
\hline SCAGR7 & 1 & 0.000 & 4 & 4.000 \\
\hline SCFXM1 & 31 & 1.667 & 31 & 16.500 \\
\hline SCFXM2 & 31 & 1.000 & 31 & 25.103 \\
\hline SCFXM3 & 31 & 0.909 & 31 & 32.212 \\
\hline SCORPION & 1 & 0.500 & 0 & 38.250 \\
\hline SCRS8 & 1 & 1.000 & 7 & 785536.364 \\
\hline SCSD1 & 1 & 0.500 & 13 & 12.500 \\
\hline SCSD6 & 1 & 0.571 & 15 & 16.429 \\
\hline SCSD8 & 1 & 0.654 & 20 & 42.904 \\
\hline SCTAP1 & 1 & 0.500 & 31 & 18.750 \\
\hline SCTAP2 & 1 & 1.310 & 28 & 124.517 \\
\hline SCTAP3 & 1 & 2.500 & 30 & 174.761 \\
\hline SHARE1B & 1 & 0.500 & 8 & 5.000 \\
\hline SHARE2B & 1 & 0.000 & 5 & 1.000 \\
\hline SHELL & 1 & 0.941 & 0 & 40.706 \\
\hline SHIP04L & 1 & 0.938 & 0 & 0.438 \\
\hline SHIP04S & 1 & 1.000 & 0 & 0.333 \\
\hline SHIP08L & 1 & 1.089 & 0 & 0.500 \\
\hline SHIP08S & 1 & 1.259 & 0 & 0.556 \\
\hline SHIP12L & 1 & 1.203 & 0 & 0.576 \\
\hline SHIP12S & 1 & 1.102 & 0 & 0.475 \\
\hline SIERRA & 0 & 0.480 & 0 & 14.960 \\
\hline STAIR & 31 & 3.900 & 1 & 5.900 \\
\hline STANDATA & 1 & 2.000 & 31 & 43.000 \\
\hline STANDGUB & 1 & 8.000 & 0 & 2.000 \\
\hline STANDMPS & 1 & 1.000 & 31 & 39.778 \\
\hline STOCFOR1 & 1 & 0.000 & 10 & 8.000 \\
\hline STOCFOR2 & 1 & 1.000 & 13 & 189.336 \\
\hline TRUSS & 1 & 0.236 & 15 & 35.163 \\
\hline TUFF & 14 & 4.286 & 0 & 0.286 \\
\hline VTP.BASE & 1 & 1.000 & 31 & 17.000 \\
\hline WOOD1P & 1 & 0.302 & 31 & 22.698 \\
\hline & 1 & 0.602 & & 61.329 \\
\hline
\end{tabular}

Table III.: Rigorous bounds for the Netlib problems using lp_solve 3.2 $\rho_{d}$ - distance to dual infeasibility, $\rho_{p}$ - distance to primal infeasibility, $f^{*}-$ lower bound, $\overline{f^{*}}-$ upper bound, $\mu$ - relative accuracy

\begin{tabular}{lrrrrr}
\hline Name & $\rho_{d}$ & $\rho_{p}$ & $\underline{f^{*}}$ & $\overline{f^{*}}$ & $\mu$ \\
\hline SC105 & 0.133484 & 0 & $-2.1696 e+13$ & $-5.2201 e+01$ & $1.0000 e-00$ \\
SC205 & 0.010023 & 0 & $-\infty$ & $-5.2201 e+01$ & $1.8125 e-05$ \\
SC50A & 0.562500 & 0 & $-5.8365 e+04$ & $-6.4574 e+01$ & $9.9889 e-01$ \\
SC50B & 0.421875 & 0 & $-7.3733 e+02$ & $-6.9999 e+01$ & $9.0506 e-01$ \\
\hline
\end{tabular}


Table IV.: Bounds for interval problems

$\rho_{d}$ - distance to dual infeasibility, $\rho_{p}$ - distance to primal infeasibility,

$f^{*}$ - lower bound, $\overline{f^{*}}$ - upper bound, $\mu$ - relative accuracy

\begin{tabular}{|c|c|c|c|c|c|}
\hline Name & $\rho_{d}$ & $\rho_{p}$ & $f^{*}$ & $\overline{f^{*}}$ & $\mu$ \\
\hline $25 \mathrm{FV} 47$ & 0 & 0 & $5.5013 e+\overline{03}$ & $\infty$ & $9.2351 e-05$ \\
\hline 80BAU3B & 0 & 0 & $9.8720 e+05$ & $9.8726 e+05$ & $6.1059 e-05$ \\
\hline ADLITTLE & 0.051651 & 0 & $2.2549 e+05$ & $\infty$ & $3.0616 e-05$ \\
\hline AFIRO & 1.000000 & 0.397390 & $-4.6476 e+02$ & $-4.6460 e+02$ & $3.4213 e-04$ \\
\hline $\mathrm{AGG} 2$ & 0.771400 & 0 & $-2.0240 e+07$ & $\infty$ & $2.7134 e-05$ \\
\hline AGG3 & 0.771400 & 0 & $1.0311 e+07$ & $\infty$ & $9.2632 e-05$ \\
\hline $\mathrm{AGG}$ & 0.771400 & 0 & $-3.5993 e+07$ & $\infty$ & $3.3187 e-05$ \\
\hline BANDM & 0.000418 & 0 & $-1.5864 e+02$ & $\infty$ & $7.2054 e-05$ \\
\hline BEACONFD & 0 & 0 & $3.3592 e+04$ & $\infty$ & $1.1010 e-05$ \\
\hline BLEND & 0.040726 & 0.003541 & $-3.0816 e+01$ & $-3.0803 e+01$ & $4.2114 e-04$ \\
\hline BNL1 & 0.106400 & 0 & $1.9775 e+03$ & $\infty$ & $8.6913 e-05$ \\
\hline BNL2 & 0 & 0 & $1.8112 e+03$ & $\infty$ & $2.2986 e-05$ \\
\hline BORE3D & 0.003539 & 0 & $1.3731 e+03$ & $\infty$ & $2.1177 e-05$ \\
\hline BRANDY & 0 & 0 & $-\infty$ & $\infty$ & $\mathrm{NaN}$ \\
\hline CAPRI & 0.095510 & 0.000252 & $2.6895 e+03$ & $2.6935 e+03$ & $1.4821 e-03$ \\
\hline CYCLE & 0 & 0 & $-\infty$ & $\infty$ & $\mathrm{NaN}$ \\
\hline CZPROB & 0.008807 & 0 & $2.1852 e+06$ & $\infty$ & $1.3316 e-05$ \\
\hline D2Q06C & 0 & 0 & $1.2278 e+05$ & $\infty$ & $4.8916 e-05$ \\
\hline D6CUBE & 2.000000 & 0 & $3.1549 e+02$ & $\infty$ & $2.0404 e-05$ \\
\hline DEGEN2 & 1.000000 & 0 & $-1.4352 e+03$ & $\infty$ & $1.0747 e-05$ \\
\hline E226 & 0 & 0 & $-1.8753 e+01$ & $\infty$ & $3.1879 e-01$ \\
\hline ETAMACRO & 0.200000 & 0 & $-7.5573 e+02$ & $\infty$ & $2.0841 e-05$ \\
\hline FFFFF 800 & 0.033046 & 0 & $5.5565 e+05$ & $\infty$ & $4.7258 e-05$ \\
\hline FINNIS & 0 & 0 & $-\infty$ & $\infty$ & $\mathrm{NaN}$ \\
\hline FIT1D & $\infty$ & 500000 & $-9.1464 e+03$ & $-9.1462 e+03$ & $1.8396 e-05$ \\
\hline FIT1P & 0.437500 & 1.271887 & $9.1463 e+03$ & $9.1476 e+03$ & $1.4385 e-04$ \\
\hline FIT2D & $\infty$ & 17.000000 & $-6.8465 e+04$ & $-6.8463 e+04$ & $1.5199 e-05$ \\
\hline FIT2P & 1.000000 & 1.057333 & $6.8464 e+04$ & $6.8469 e+04$ & $8.6123 e-05$ \\
\hline GANGES & 1.000000 & 0 & $-1.0960 e+05$ & $\infty$ & $1.6019 e-04$ \\
\hline GFRD-PNC & 0.347032 & 0 & $6.9018 e+06$ & $\infty$ & $5.7493 e-05$ \\
\hline GREENBEA & 0 & 0 & $-\infty$ & $\infty$ & $\mathrm{NaN}$ \\
\hline GREENBEB & 0 & 0 & $-\infty$ & $\infty$ & $\mathrm{NaN}$ \\
\hline GROW15 & 0.968073 & 0.572842 & $-1.0687 e+08$ & $-1.0687 e+08$ & $1.6424 e-05$ \\
\hline GROW 22 & 0.968073 & 0.572842 & $-1.6084 e+08$ & $-1.6083 e+08$ & $1.7269 e-05$ \\
\hline GROW7 & 0.968073 & 0.572842 & $-4.7788 e+07$ & $-4.7787 e+07$ & $1.6718 e-05$ \\
\hline ISRAEL & 0.166850 & 0.02 & $-8.9665 e+05$ & $-8.9664 e+05$ & $1.8453 e-05$ \\
\hline KB2 & 0.018802 & 0.00 & $-1.7499 e+03$ & $-1.7498 e+03$ & $1.0083 e-04$ \\
\hline LOTFI & 0 & 0.000306 & $-\infty$ & $-2.5254 e+01$ & $4.1965 e-04$ \\
\hline MAROS & 0 & 0 & $-\infty$ & $\infty$ & $\mathrm{NaN}$ \\
\hline MAROS-R7 & 0.628096 & 1.000000 & $1.4972 e+06$ & $1.4973 e+06$ & $6.3798 e-05$ \\
\hline MODSZK1 & 0.108469 & 0 & $2.7040 e+02$ & $\infty$ & $1.6995 e-01$ \\
\hline PEROLD & 0.000943 & 0 & $-\infty$ & $\infty$ & $\mathrm{NaN}$ \\
\hline PILOT4 & 0.000075 & 0 & $-2.5813 e+03$ & $\infty$ & $7.4535 e-05$ \\
\hline PILOT 87 & 0 & 0 & $3.0170 e+02$ & $\infty$ & $1.8799 e-05$ \\
\hline PILOT.JA & 0.000750 & 0 & $-6.1134 e+03$ & $\infty$ & $5.1024 e-05$ \\
\hline PILOTNOV & 0.000750 & 0 & $-4.4974 e+03$ & $\infty$ & $3.3681 e-05$ \\
\hline PILOT.WE & 0.044874 & 0 & $-2.7204 e+06$ & $\infty$ & $1.2197 e-04$ \\
\hline QAP8 & 4.000000 & 0 & $-\infty$ & $\infty$ & $\mathrm{NaN}$ \\
\hline RECIPE & 0 & 0 & $-2.6662 e+02$ & $\infty$ & $2.3744 e-05$ \\
\hline SC105 & 0.133484 & 0 & $-5.2205 e+01$ & $-5.2199 e+01$ & $1.2454 e-04$ \\
\hline $\mathrm{SC} 205$ & 0.010023 & 0 & $-5.2206 e+01$ & $\infty$ & $7.3685 e-05$ \\
\hline SC50A & 562500 & 0 & $-6.4578 e+01$ & $-6.4573 e+01$ & $7.5860 e-05$ \\
\hline SC50B & 0.421875 & 0 & $-7.0003 e+01$ & $-6.9998 e+01$ & $7.7140 e-05$ \\
\hline SCAGR 25 & 0.034646 & 0.021077 & $-1.4754 e+07$ & $-1.4752 e+07$ & $1.1701 e-04$ \\
\hline SCAGR7 & 0.034646 & 0.022644 & $-2.3315 e+06$ & $-2.3312 e+06$ & $1.2282 e-04$ \\
\hline SCFXM1 & 0 & 0 & $-\infty$ & $\infty$ & $\mathrm{NaN}$ \\
\hline SCFXM2 & 0 & 0 & $-\infty$ & $\infty$ & $\mathrm{NaN}$ \\
\hline SCFXM3 & 0 & 0 & $-\infty$ & $\infty$ & $\mathrm{NaN}$ \\
\hline SCORPION & 0.949393 & 0 & $1.8781 e+03$ & $\infty$ & $2.9037 e-05$ \\
\hline SCRS8 & 0 & 0 & $9.0426 e+02$ & $\infty$ & $3.7130 e-05$ \\
\hline SCSD1 & 000 & 57 & $8.6665 e+00$ & +00 & $1.4261 e-04$ \\
\hline SCSD6 & 1.000000 & 1.603351 & $5.0499 e+01$ & $5.0506 e+01$ & $1.4791 e-04$ \\
\hline SCSD 8 & 1.000000 & 0.268363 & $9.0494 e+02$ & $9.0550 e+02$ & $6.2245 e-04$ \\
\hline SCTAP1 & 1.000000 & 0.032258 & $1.4122 e+03$ & $1.4125 e+03$ & $2.2764 e-04$ \\
\hline SCTAP2 & 1.000000 & 0.586563 & $1.7248 e+03$ & $1.7260 e+03$ & $6.8090 e-04$ \\
\hline SCTAP3 & 1.000000 & 0.381250 & $1.4240 e+03$ & $\infty$ & $1.1257 e-05$ \\
\hline SHARE1B & 0.000751 & 0.000015 & $-7.6602 e+04$ & $-7.6428 e+04$ & $2.2704 e-03$ \\
\hline SHARE2B & 0.287893 & 0.001747 & $-4.1584 e+02$ & $-4.1559 e+02$ & $6.1583 e-04$ \\
\hline SHELL & 1.777778 & 0 & $1.2088 e+09$ & $\infty$ & $1.4568 e-05$ \\
\hline SHIP04L & 13.146000 & 0 & $1.7933 e+06$ & $\infty$ & $1.0788 e-05$ \\
\hline SHIP04S & 13.146000 & 0 & $1.7987 e+06$ & $\infty$ & $1.1168 e-05$ \\
\hline SHIP08L & 21.210000 & 0 & $1.9090 e+06$ & $\infty$ & $1.1798 e-05$ \\
\hline
\end{tabular}


Computational Experience with Rigorous Error Bounds for the Netlib lp Library 13

\begin{tabular}{|c|c|c|c|c|c|}
\hline Name & $\rho_{d}$ & $\rho_{p}$ & $f^{*}$ & $\overline{\overline{f^{*}}}$ & $\mu$ \\
\hline SHIP08S & 21.210000 & 0 & $\overline{1.9201 e+\overline{06}}$ & $\infty$ & $1.2431 e-05$ \\
\hline SHIP12L & 7.434000 & 0 & $1.4702 e+06$ & $\infty$ & $1.3344 e-05$ \\
\hline SHIP12S & 7.434000 & 0 & $1.4892 e+06$ & $\infty$ & $1.5090 e-05$ \\
\hline SIERRA & $\infty$ & 0 & $1.5376 e+07$ & $\infty$ & $1.1848 e-03$ \\
\hline STAIR & 0 & 0.000580 & $-\infty$ & $-2.5126 e+02$ & $3.7820 e-05$ \\
\hline STANDATA & 1.000000 & 0 & $1.2577 e+03$ & $\infty$ & $1.3912 e-05$ \\
\hline STANDGUB & 0 & 0 & $1.2577 e+03$ & $\infty$ & $1.3912 e-05$ \\
\hline STANDMPS & 1.000000 & 0 & $1.4060 e+03$ & $\infty$ & $1.4991 e-05$ \\
\hline STOCFOR1 & 0.011936 & 0.001203 & $-4.1134 e+04$ & $-4.1126 e+04$ & $1.8826 e-04$ \\
\hline STOCFOR2 & 0.000064 & 0.000437 & $-3.9026 e+04$ & $\infty$ & $4.4179 e-05$ \\
\hline TRUSS & 10.000000 & 0.518928 & $4.5877 e+05$ & $4.5910 e+05$ & $7.1544 e-04$ \\
\hline TUFF & 0.017485 & 0 & $2.9213 e-01$ & $\infty$ & $1.9790 e-05$ \\
\hline VTP.BASE & 0.500000 & 0 & $1.2982 e+05$ & $\infty$ & $5.9034 e-05$ \\
\hline WOOD1P & 1.000000 & 0 & $1.4428 e+00$ & $\infty$ & $4.7868 e-05$ \\
\hline WOODW & 1.000000 & 0 & $1.3044 e+00$ & $\infty$ & $2.6084 e-05$ \\
\hline
\end{tabular}

Table V.: Performance of the interval bounds

$i_{f *}$ - iterations to compute lower bound,

$\overline{t_{f^{*}}}$ - time to compute lower bound, $t_{f^{*}}$ - time to compute approximate solution,

$i \overline{\overline{f *}}-$ iterations to compute upper bound,

$t \frac{f^{*}}{f^{*}}$-time to compute upper bound

\begin{tabular}{|c|c|c|c|c|}
\hline Name & $i_{f^{*}}$ & $t_{f *} / t_{f} *$ & $i \overline{f^{*}}$ & $t_{f^{*}} / t_{f^{*}}$ \\
\hline $25 \mathrm{FV} 47$ & $\overline{\overline{1}}$ & 0.107 & 0 & 0.030 \\
\hline 80BAU $3 B$ & 1 & 1.921 & 1 & 0.393 \\
\hline ADLITTLE & 1 & & 31 & \\
\hline AFIRO & 1 & & 9 & \\
\hline AGG2 & 1 & 0.750 & 31 & 3.250 \\
\hline AGG3 & 1 & 0.750 & 31 & 3.250 \\
\hline AGG & 1 & 0.667 & 2 & 2880000.000 \\
\hline BANDM & 1 & 0.273 & 31 & 14.455 \\
\hline BEACONFD & 1 & 0.500 & 9 & 8640050.000 \\
\hline BLEND & 1 & 0.000 & 9 & 1.000 \\
\hline BNL1 & 1 & 0.517 & 0 & 3.172 \\
\hline BNL2 & 1 & 1.809 & 31 & 87.787 \\
\hline BORE3D & 1 & 0.333 & 0 & 6.667 \\
\hline BRANDY & 31 & 1.333 & 0 & 0.333 \\
\hline CAPRI & 3 & 1.000 & 8 & 7.250 \\
\hline CYCLE & 19 & 11.365 & 0 & 1.670 \\
\hline CZPROB & 1 & 0.420 & 31 & 40.407 \\
\hline $\mathrm{D} 2 \mathrm{Q} 06 \mathrm{C}$ & 1 & 0.283 & 31 & 15.738 \\
\hline D6CUBE & 1 & 0.360 & 0 & 7.500 \\
\hline DEGEN2 & 1 & 0.152 & 0 & 1.818 \\
\hline E226 & 1 & 0.200 & 31 & 4.000 \\
\hline ETAMACRO & 1 & 0.417 & 0 & 5.750 \\
\hline FFFFF 800 & 1 & 0.421 & 3 & 454752.632 \\
\hline FINNIS & 31 & 2.625 & 2 & 1.250 \\
\hline FIT1D & 0 & 0.000 & 5 & 0.250 \\
\hline FIT1P & 1 & 0.340 & 2 & 35.887 \\
\hline FIT2D & 0 & 0.005 & 4 & 0.028 \\
\hline FIT2P & 1 & 0.728 & 2 & 158.333 \\
\hline GANGES & 1 & 0.889 & 31 & 588.844 \\
\hline GFRD-PNC & 1 & 0.917 & 9 & 153.417 \\
\hline GREENBEA & 31 & 0.391 & 0 & 9.666 \\
\hline GREENBEB & 31 & 0.434 & 0 & 10.398 \\
\hline GROW15 & 1 & 0.033 & 2 & 3.083 \\
\hline GROW 22 & 1 & 0.059 & 2 & 6.812 \\
\hline GROW7 & 1 & 0.200 & 2 & 2.800 \\
\hline ISRAEL & 1 & 0.500 & 1 & 0.000 \\
\hline KB2 & 1 & & 4 & \\
\hline LOTFI & 31 & 1.500 & 2 & 3.500 \\
\hline MAROS & 31 & 0.505 & 0 & 2.218 \\
\hline MAROS-R7 & 1 & 0.404 & 2 & 64.692 \\
\hline MODSZK1 & 11 & 5.688 & 0 & 19.500 \\
\hline PEROLD & 13 & 4.159 & 31 & 14.732 \\
\hline PILOT4 & 7 & 1.250 & 31 & 11.917 \\
\hline PILOT87 & 1 & 0.073 & 31 & 0.711 \\
\hline PILOT.JA & 7 & 0.582 & 0 & 0.365 \\
\hline PILOTNOV & 1 & 0.436 & 0 & 1.103 \\
\hline PILOT.WE & 6 & 0.337 & 18 & 29697.938 \\
\hline QAP8 & 18 & 753.096 & 0 & 0.088 \\
\hline RECIPE & 0 & & 0 & \\
\hline $\mathrm{SC} 105$ & 1 & & 6 & \\
\hline
\end{tabular}




\begin{tabular}{lrrrr}
\hline Name & $i_{f^{*}}$ & $t_{f^{*} / t_{f^{*}}}$ & $i_{f^{*}}$ & \multicolumn{1}{c}{$f_{f^{*}} / t_{f^{*}}$} \\
\hline SC205 & 1 & 0.500 & 31 & 8.500 \\
SC50A & 1 & & 6 & \\
SC50B & 1 & & 2 & \\
SCAGR25 & 1 & 0.308 & 5 & 18.615 \\
SCAGR7 & 1 & 0.000 & 3 & 3.000 \\
SCFXM1 & 31 & 1.167 & 31 & 8.333 \\
SCFXM2 & 31 & 0.621 & 31 & 12.655 \\
SCFXM3 & 31 & 0.478 & 31 & 17.567 \\
SCORPION & 1 & 0.500 & 0 & 26.750 \\
SCRS8 & 1 & 1.000 & 31 & 104.000 \\
\hline SCSD1 & 1 & 0.500 & 12 & 11.500 \\
SCSD6 & 1 & 0.714 & 12 & 14.857 \\
SCSD8 & 1 & 4.635 & 14 & 33.923 \\
SCTAP1 & 1 & 0.750 & 9 & 7.000 \\
SCTAP2 & 1 & 1.407 & 9 & 65.370 \\
SCTAP3 & 1 & 2.667 & 31 & 104.622 \\
SHARE1B & 1 & 0.500 & 8 & 4.000 \\
SHARE2B & 1 & 0.000 & 6 & 1.000 \\
SHELL & 1 & 1.125 & 0 & 62.500 \\
SHIP04L & 1 & 0.941 & 0 & 0.353 \\
\hline SHIP04S & 1 & 0.917 & 0 & 0.333 \\
SHIP08L & 1 & 1.125 & 0 & 0.482 \\
SHIP08S & 1 & 1.214 & 0 & 0.536 \\
SHIP12L & 1 & 1.239 & 0 & 0.769 \\
SHIP12S & 1 & 1.049 & 0 & 0.508 \\
SIERRA & 0 & 0.480 & 0 & 14.320 \\
STAIR & 31 & 3.300 & 6 & 9.400 \\
STANDATA & 1 & 7.000 & 31 & 80.000 \\
STANDGUB & 1 & 4.000 & 0 & 1.000 \\
STANDMPS & 1 & 1.250 & 31 & 23.625 \\
\hline STOCFOR1 & 1 & 0.000 & 15 & 4.000 \\
STOCFOR2 & 1 & 1.076 & 31 & 211.750 \\
TRUSS & 1 & 0.200 & 11 & 29.779 \\
TUFF & 12 & 4.286 & 0 & 0.429 \\
VTP.BASE & 1 & 0.500 & 1 & 0.500 \\
WOOD1P & 1 & 0.333 & 0 & 8.405 \\
WOODW & 1 & 0.531 & 3 & 46.261 \\
\hline & & & & \\
& & & & \\
\hline
\end{tabular}

\section{References}

1. Alefeld, G. and J. Herzberger: 1983, Introduction to Interval Computations. New York: Academic Press.

2. Beeck, H.: 1978, 'Linear Programming with Inexact Data'. Technical Report 7830, Abteilung Mathematik, TU München.

3. Berkelaar, M., P. Notebaert, and K. Eikland, 'lp_solve'. World Wide Web. http://groups . yahoo. com/group/lp_solve.

4. Floudas, C.: 2000, Deterministic Global Optimization - Theory, Methods and Applications, Vol. 37 of Nonconvex Optimization and Its Applications. Dordrecht, Boston, London: Kluwer Academic Publishers.

5. Fourer, R. and D. M. Gay: 1994, 'Experience with a Primal Presolve Algorithm'. In: W. W. Hager, D. W. Hearn, and P. M. Pardalos (eds.): Large Scale Optimization: State of the Art. Norwell, MA, USA, and Dordrecht, The Netherlands: Kluwer Academic Publishers Group, pp. 135-154.

6. Free Software Foundation, 'gcc'. http://gcc.gnu.org.

7. Hansen, E. and G. W. Walster: 2003, Global Optimization Using Interval Analysis, Pure and Applied Mathematics. Dekker, second edition edition.

8. Jansson, C.: 2004a, 'A rigorous lower bound for the optimal value of convex optimization problems'. J. Global Optimization 28, 121-137.

9. Jansson, C.: 2004b, 'Rigorous Lower and Upper Bounds in Linear Programming'. SIAM J. Optim. 14(3), 914-935.

10. Kearfott, R.: 1996, Rigorous Global Search: Continuous Problems. Dordrecht: Kluwer Academic Publisher. 
11. Keil, C.: 2004, 'LURUPA - Rigorose Fehlerschranken für Lineare Programme'. Diplomarbeit, Technische Universität Hamburg-Harburg.

12. Knüppel, O.: 1998, 'PROFIL/BIAS and extensions, Version 2.0'. Technical report, Inst. f. Informatik III, Technische Universität Hamburg-Harburg.

13. Krawczyk, R.: 1975, 'Fehlerabschätzung bei linearer Optimierung'. In: K. Nickel (ed.): Interval Mathematics, Vol. 29 of Lecture Notes in Computer Science. Berlin: Springer Verlag, pp. 215-222.

14. Moore, R.: 1979, Methods and Applications of Interval Analysis. Philadelphia: SIAM.

15. Netlib, 'Netlib Linear Programming Library'. http://www.netlib.org/lp.

16. Neumaier, A.: 1990, Interval Methods for Systems of Equations, Encyclopedia of Mathematics and its Applications. Cambridge University Press.

17. Neumaier, A.: 2001, Introduction to Numerical Analysis. Cambridge University Press.

18. Neumaier, A.: 2004, 'Complete Search in Continuous Global Optimization and Constraint Satisfaction'. Acta Numerica 13, 271-369.

19. Neumaier, A. and O. Shcherbina: 2004, 'Safe bounds in linear and mixed-integer programming'. Mathematical Programming, Ser. A 99, 283-296.

20. Ordóñez, F. and R. Freund: 2003, 'Computational experience and the explanatory value of condition measures for linear optimization'. SIAM J. Optimization 14(2), 307-333.

21. Rump, S.: 1983, 'Solving Algebraic Problems with High Accuracy. Habilitationsschrift'. In: U. Kulisch and W. Miranker (eds.): A New Approach to Scientific Computation. New York: Academic Press, pp. 51-120.

Address for Offprints:

Technische Informatik III

Technische Universität Hamburg-Harburg

Schwarzenbergstraße 95, 21073 Hamburg 
netlib_experience_5.5.tex; 4/07/2005; $10: 40 ;$ p.16 